\title{
La memoria en el espacio: cartografías del gueto de Varsovia*
}

\author{
Memory in space: \\ cartography of the Warsaw ghetto \\ A memoria no espaço: \\ cartografias do gueto de Varsóvia
}

Fecha de entrega: 10 de febrero de 2012

Fecha de evaluación: 27 de abril de 2012

Fecha de aprobación: 13 de junio de 2012

Pamela Colombo ${ }^{* *}$

\section{Resumen}

En este artículo se problematiza la relación entre espacio, tiempo y memoria, a partir de la puesta en diálogo de los desarrollos teóricos en torno al espacio relacional que ha realizado David Harvey y los estudios sobre rememoración de Walter Benjamin. Se tomará la obra de teatro

* Este artículo es resultado de las investigaciones de la autora en el grupo "La Filosofía después del Holocausto: Vigencia de sus Lógica Perversas" en el Instituto de Filosofía del Centro de Ciencias Humanas y Sociales. De igual forma, está vinculado a su investigación de tesis doctoral sobre el tiempo y el espacio en los procesos de desaparición forzada.

* Doctoranda en el Instituto de Filosofía del Centro de Ciencias Humanas y Sociales de Madrid (España). Licenciada en Sociología por la Universidad de Buenos Aires. Máster en Filosofia de la Historia de la Universidad Autónoma de Madrid. Ha realizado estancias de investigación en la Albert-Ludwigs Universität Freiburg, en la Freie Universität Berlin, en el Ibero-Amerikanische Institut Berlin y en la City University of New York. Correo electrónico: pamela.colombo@cchs.csic.es 
de Juan Mayorga: El cartógrafo. Varsovia, 1:400.000 como disparadora de estas problemáticas.

Palabras clave: espacio, memoria, David Harvey, Walter Benjamin, lugar, gueto.

\section{Abstract}

This article presents the problem in the relationship between space, time and memory, from the dialogue of theoretical developments regarding relational space that has performed by David Harvey and Walter Benjamin's studies on rememoration. The play by Juan Mayorga: The cartographer. Warsaw, 1:400.000 will be taken as a trigger of these problems.

Keywords: space, memory, David Harvey, Walter Benjamin, place, ghetto.

\section{Resumo}

Neste artigo problematiza-se a relação entre espaço, tempo e memória, desde o inicio de diálogo dos desenvolvimentos teóricos em torno ao espaço relacional que tem realizado o David Harvey e os estudos sobre a relembrança de Walter Benjamin. Tomar-se-á a peça de teatro do Juan Mayorga: O cartógrafo. Varsóvia, 1:400.000 como desencadeamento destas problemáticas.

Palavras-chave: espaço, memória, David Harvey, Walter Benjamin, lugar, gueto.

\section{Introducción}

La obra de Juan Mayorga El cartógrafo. Varsovia, 1:400.000 comienza situándonos en un lugar: Varsovia; y está enmarcada - en apariencia- en dos momentos cronológicamente separados: la Varsovia ocupada por los nazis y la Varsovia actual. Blanca es la esposa de un diplomático que fue trasladado a Varsovia por razones de trabajo. A los pocos días de llegar, Blanca pareciera haber encontrado un propósito 
para emplear su tiempo libre en esta nueva ciudad: hacer coincidir las imágenes del gueto de Varsovia - que ha visto por azar en una muestra fotográfica dentro de una sinagoga - con la Varsovia actual. Con estas imágenes del pasado a cuestas, Blanca comienza a deambular por la ciudad. Su primera búsqueda - la primera entre muchas - consistirá en hacer coincidir los lugares de las fotos con los lugares de la Varsovia actual, y así ir viendo lo que falta y lo que sobrevivió a aquella época. En este deambular entre calles y anécdotas, Blanca conocerá la historia del cartógrafo y la niña: se dice que un viejo cartógrafo y una niña realizaron un mapa del gueto mientras vivían allí confinados. El mapa (real o imaginado) que construyeron la niña y el cartógrafo puede pensarse como un intento por darle una imagen a lo que se sabe que desaparecerá. Darle presencia a lo que irremediablemente se volverá ausencia. Hacer un mapa como gesto, para darle un lugar al gueto, a la representación del gueto.

Un tema que atraviesa constante la obra es, justamente, esta necesidad imperiosa de situar, de dar un lugar al horror. Representar para poder dar cuenta de la "sombra", para resituar la falta. A medida que avanza un poco la trama, la atención ya no se detiene solamente sobre el punto de vista de la comunidad que ha sido confinada dentro del gueto, sino que por el contrario Mayorga trae el tema del afuera. Y junto con el afuera del gueto, aparecen otros actores: los nazis, los ciudadanos no judíos de Varsovia... Este traer a escena, mostrar otros puntos de vista sobre el gueto, "abre" sin dudas la percepción que hasta ese momento teníamos de su espacio; es decir, podemos ver cómo emergen remisiones a territorios diferentes: los judíos de adentro (los sitiados), los perpetradores nazis (los sitiadores) y los pobladores comunes de Varsovia (y estos, a su vez, interpretan diferentes papeles: pueden ser espectadores, partícipes o cómplices).

Hay un lugar, Varsovia, y múltiples puntos de vista del gueto que lo hacen cada vez un espacio diferente. De esta manera vemos cómo el espacio del gueto se relativiza. Tomar conciencia de que el espacio es relativo es tomar conciencia de que la generación de conocimiento en relación con el espacio depende del punto de vista de quien lo produce. El espacio del gueto no es el mismo para aquellos que lo concibieron en sus cabezas, para aquellos que lo experimentaron o para aquellos que desarrollaron su vida cotidiana fuera del gueto.

Las escenas se dividen, al menos al comienzo, entre la Varsovia actual y las escenas con el cartógrafo y la niña en la época del gueto. Estos dos tiempos: el del gueto y el del ahora, en un principio parecerían ser los tiempos sobre los que se estructurará 
la obra; pero a medida que la historia avanza, comienzan a aflorar otros múltiples tiempos, que remiten a diferentes momentos históricos de la propia Varsovia. A su vez, simultáneamente — en la Varsovia actual- los personajes también experimentarán la emergencia y superposición de otros espacios ajenos a Varsovia. Podríamos preguntarnos, entonces: ¿de qué modo la memoria se inscribe en el espacio? ¿La pregunta por Varsovia es la pregunta por la memoria que conserva ese espacio? ¿O acaso no es también la pregunta por lo que ese espacio provoca, por las memorias que ese espacio dispara? ¿Cuántas Varsovias y cuántos guetos hay? ¿Quién recuerda en el espacio: el sujeto o la ciudad?

Tomando la obra de Mayorga como disparadora de estas problemáticas, el objetivo central de este artículo radica en poner en diálogo las reflexiones en torno al espacio relacional realizadas por Harvey, junto con los desarrollos teóricos sobre la rememoración de Benjamin. Propongo ese diálogo teórico porque considero que puede enriquecer el modo en que nos aproximamos a la compleja relación entre espacio, tiempo y memoria.

En el primer apartado desagrego la conceptualización de espacio relacional que sistematiza Harvey en varios de sus trabajos. A partir de ello, propongo una triada para distinguir analíticamente distintas maneras en que la memoria puede inscribirse en el espacio. En el segundo apartado retomo los desarrollos de Benjamin en torno a los procesos de rememoración y planteo que el aspecto espacial en la teoría benjaminiana reside en que la memoria tiene la potencialidad de actualizar de manera diferida y dislocante espacios y tiempos perimidos en un mismo lugar.

\section{La permanencia transitoria de Varsovia}

En el texto Space as a keyword, Harvey (2006) sistematiza la descripción de la siguiente triada: espacio absoluto, espacio relativo y espacio relacional. Cada conceptualización de esta triada refiere representaciones del espacio que han tenido (y tienen) un papel central en la sociedad occidental. El espacio absoluto está ligado, sobre todo, a las teorizaciones de Newton y Descartes, que presentan el espacio como algo preexistente, que se nos ofrece como una red inmóvil en la cual estandarizar medidas y hacer cálculos; geométricamente hablando estaríamos frente al espacio de Euclides. El espacio relativo se sustenta especialmente a partir de la teoría de la relatividad de Einstein. Dicha teoría es la que muestra cómo es posible que haya diversas geometrías entre las 
cuales elegir, y que el marco espacial desde el que se produce la aproximación también depende de lo que esté siendo relativizado y por quién. Por último estaría el espacio relacional, que es la conceptualización que retomaré en este artículo (Harvey, 2006) ${ }^{1}$.

La noción de espacio relacional que expone Harvey se sustenta, sobre todo, a partir de los desarrollos teóricos de Leibniz y Whitehead (Murdoch, 2006; Harvey, 1996). De Leibniz retoma la idea de que el espacio (space) y el lugar (place) se constituyen a partir de los procesos y substancias que los atraviesan ${ }^{2}$. De Whitehead retomará la

1 Harvey habla de mantener los tres conceptos de espacio (absoluto, relativo y relacional) en una "tensión dialéctica". Para clarificar un poco estos puntos, me referiré aqui al ejemplo que Harvey utiliza para demostrar cómo existen estos tres espacios de manera simultánea. El ejemplo se sitúa en el marco de una conferencia. El espacio absoluto seria aquel en que se lleva a cabo la charla, donde las palabras del orador rebotan contra los muros físicos. El tiempo absoluto sería aquel que marca si uno llega o no tarde a la conferencia, es decir, "si llega a tiempo" o no para escuchar la conferencia. El espacio relativo sería aquel que depende del punto exacto de cada uno de los que están escuchando la charla. Y como cada persona está en una distancia espacio-temporal distinta, la experiencia de la charla será diferente. El espacio-tiempo relacional que emerge en la charla dependerá de lo que cada uno de nosotros está pensando durante la misma; es decir, los espacios y tiempos otros que estamos trayendo al aqui y ahora. Esta superposición que atraviesa el espacio hace que ese espacio absoluto se cargue de otras muchas cosas. "The decision to use one or other conception certainly depends on the nature of the phenomena under investigation. The absolute conception may be perfectly adequate for issues of property boundaries and border determinations but it helps me not a whit with the question of what is Tiananmen Square, Ground Zero or the Basilica of Sacré-Cœur" (Harvey, 2006, p. 275). Es importante hacer la salvedad de que existe una tensión dialéctica entre el espacio absoluto, relativo y relacional. Al hacer hincapié en el espacio relacional no pretendo ponderar un espacio sobre otro, ya que todos estos espacios se dan cita a la vez; sino que el énfasis sobre esta conceptualización tiene su razón de ser por el tipo de análisis que se pretende realizar aqui: "I find it far more interesting in principle to keep the three concepts in dialectical tension with each other and to think constantly through the interplay among them. Ground Zero is an absolute space at the same time as it is relative and relational in space-time" (Harvey, 2006, p. 276).

2 "If we accept the transformation I am here proposing and drop the theological idea of a singular creative deity and accept social choice and creativity with respect to the orderings of space and time, then the issue of how these different social choices are arrived at and whether or not they are embedded in entirely separate worlds of sensations and incommunicable language (as opposed to the multiplication of perspectives on a single universe) becomes central [...] there is a singular actually existing framework of space and time and that social variations arise only by virtue of our varied perspectives [...] on a common universe [...] Such possible worlds are not entirely outside of human experience, though Leibniz might reasonable claim that in some sense the worlds they depict are outside of the material world of sense perception [...] A secularized and social understanding of relational space and time on a Leibnizian foundation provides, in short, a strong theoretical and logical framework capable of representing the variegated social practices of construction of space and time [...] It also permits the exploration of alternative modes of production in terms of the production of different possible worlds of spatio-temporality" (Harvey, 1996, pp. 254-255). 
idea de que esos procesos están constituidos por relaciones ${ }^{3}$ (Harvey, 1996; Murdoch, 2006). Harvey (2006) agregará que los lugares llegan a constituirse como tales en la medida en que logran sonsacarle suerte de "estabilizaciones" al fluir de esos diferentes procesos; dichas estabilizaciones las llamará "permanencias". Estas, por más sólidas que parezcan, son siempre contingentes, ya que son parte de un proceso en el que se crean y se sostienen, pero en el que posteriormente también cabe la posibilidad de que se disuelvan ${ }^{4}$. Esta aproximación posibilita pensar los lugares como resultado de "configuraciones dinámicas de relativa permanencia". En resumidas cuentas, las permanencias no son eternas; la creación de lugares (place formation) no queda nunca por fuera de la influencia del tiempo:

Is no such thing as space or time outside of the processes that define them $[\ldots]$ The relational notion of space-time implies the idea of internal relations; external influences get internalized in specific processes or things through time [...] An event or a thing at a point in space cannot be understood by appeal to what exists only at that point. It depends upon everything else going on around it [...] A wide variety of disparate influences swirling over space in the past, present and future concentrate and congeal at a certain point (e.g. within a conference room) to define the nature of that point (Harvey, 2006, p. 273).

En relación con este tema, Murdoch (2006) hace una apreciación importante, ya que remarca que no todas las relaciones que atraviesan un espacio lograrán plasmarse como permanencias. Es por ello que el espacio relacional debe pensarse de manera simultánea como resultado del consenso, pero también de la disputa, ya que para que un tipo de entramado de relaciones sea central para la producción de un lugar, debió

3 "Whitehead argued that we ought no to consider physical bodies as if they are first in space and then act upon each other. Bodies are in space, rather, only because they interact, so that space is 'only the expression of certain properties of their interaction' (Lowe), Space and time are not, therefore, independent realities, but relations derived from processes and events" (Harvey, 1996, p. 256).

4 "Entities achieve relative stability in their bounding and their internal ordering of processes creating space, for a time. Such permanences come to occupy a piece of a space in an exclusive way (for a time) and thereby define a place -their place- (for a time). The process of place formation is a process of carving out 'permanences' from the flow processes creating spaces. But the 'permanences' -no matter how solid they may seem- are not eternal: they are always subject to time as 'perpetual perishing'. They are contingent on the processes that create, sustain and dissolve them" (Harvey, 1996, p. 261). 
previamente imponerse sobre otros tipos de relaciones 5 . Tomando el caso de la ciudad de Varsovia, el espacio que resultó de la segregación y posterior desaparición física de los judíos (y, por consiguiente, del entramado de relaciones que dicha comunidad construía y sostenía) produjo un lugar concreto. El lugar Varsovia del que podemos tener experiencia hoy es la "condensación y coagulación en un punto" de diferentes relaciones (relaciones que se han impuesto por sobre otras). La "cristalización", por lo tanto, de estos entramados de relaciones en un lugar producen unas permanencias determinadas y no otras. Estas, igualmente, no son más que consolidaciones de relativa continuidad, como dirá Harvey; es decir, permanencias que eventualmente se disolverán y darán lugar a la aparición de otras nuevas. Pero hay otro factor indisociable que no puede dejarse de lado al problematizar el espacio relacional: el tiempo.

Llegados a este punto, Harvey agrega que el análisis de dichas permanencias no puede circunscribirse solamente a lo que existe en un determinado momento, sino que se debe tomar también en consideración una gran variedad de influencias que atraviesan ese espacio, y esas influencias dan cuenta de otros tiempos además del mero presente (influencias que pueden también haberse condensado en ese lugar en particular). De esta última apreciación se puede entrever claramente por qué Harvey deriva de la perspectiva del espacio relacional la necesidad de incluir en el análisis el rol que juegan las memorias colectivas en relación con la conformación de lugares (desde otras concepciones, como las del espacio absoluto o relativo, sería francamente complicado realizar dicha aproximación $)^{6}$.

5 "Some attention must be paid to spaces that do not emerge, to the sets of relations that fail to gain any kind of spatial coherence. Relations between relations therefore become important. The shape of space can be seen as the 'expression' of 'underlying' relations; but it can also be seen as the suppression of all those other relations that might have gained some amount of permanence had they not 'flickered out of existence' in Thrift's telling phrase. The relational making of space is both a consensual and contested process. 'Consensual' because relations are usually made out of agreements or alignments between two or more entities; 'contested' because the construction of one set of relations may involve both the exclusion of some entities (and their relations) as well as the forcible enrolment of others. In short, relational space is a 'power-filled' space in which some alignments come to dominate, at least for a period of time, while others come to be dominated. So while multiple sets of relations may well co-exist, there is likely to be some competition between these relations over the composition of particular spaces and places" (Murdoch, 2006, p. 20).

6 "But why and how would I, as a working geographer, find the relational mode of approaching spacetime useful? The answer is quite simply that there are certain topics, such as the political role of collective memories in urban processes, that can only be approached in this way. I cannot box political and collective memories in some absolute space (clearly situate them on a grid or a map) nor can I understand their circulation according to the rules, however sophisticated, of relative space-time. If 
Retomando lo expuesto hasta aquí, propondré un modo de desagregar de manera analítica la relación entre el espacio y la memoria. La desagregación que propongo se estructura en tres componentes ${ }^{7}$ :

1. Por un lado existe la memoria que el espacio conserva materialmente en las marcas que constituyen el paisaje actual.

2. También está la memoria que se busca "imprimir" o "sostener" de manera deliberada en el espacio. En este nivel sobre todo podríamos pensar en los procesos de monumentalización y/o musealización de la memoria.

3. Asimismo, la memoria que trae vivencias de otros tiempos y de otros espacios al presente de un lugar en particular.

Desagregaré a continuación la triada propuesta. La primera dimensión del análisis se centra en el modo en que los acontecimientos se inscriben en el espacio, esto es, cómo trazan sus historias en el espacio. Las marcas de otros tiempos y espacios ya perimidos sobreviven como restos en el presente. Podríamos pensar este nivel como aquel donde la materialidad que ha sido inscrita por el acontecimiento es el "soporte" y/o "catalizador" material de diferentes procesos de rememoración.

¿Acaso el gueto de Varsovia ha desaparecido por completo sin dejar marcas? Y la rebelión de los judíos confinados dentro del gueto, ¿dónde se ha inscrito? ¿Es posible pensar en un traslado masivo de personas - como el que se produjo en Varsovia- sin que el espacio material se llene de cicatrices? La segregación de los judíos en el gueto de Varsovia, su posterior deportación a los campos de concentración y exterminio y, finalmente, la destrucción casi total de los edificios del gueto dejan como resultado a una Varsovia arrasada, pero llena de señales.

El arrasamiento se produce en varios aspectos. Uno de ellos refiere a la desaparición física -materialmente objetiva- de los sujetos, de sus espacios de circulación.

I ask what Tiananmen Square or 'Ground Zero' mean, then the only way I can seek an answer is to think in relational terms" (Harvey, 2006, pp. 274-275).

7 Para trazar esta triada, me he inspirado claramente en la triada espacial desarrollada por Lefebvre (1991), donde distingue entre el espacio experimentado, el espacio concebido y el espacio vivido. Me limito meramente a hacer alusión al trabajo de Lefebvre, ya que entrar en el detalle de su obra es un trabajo que excede ampliamente los límites del presente artículo. 
Varsovia carga desde ese momento con la falta. Varsovia cambia a partir de esa falta. Pero Varsovia también está llena de marcas que señalan esa(s) ausencia(s). Es la memoria de lo ausente que persiste en la materia:

Blanca: Seguí por aquí, por Novolipie, hasta aquí, donde se cruza con Karmelicka. Una de las fotos decía que ahí empezó la rebelión, pero no hay señal de ello, al menos yo no vi señal. En este parque, aquí sí hay un gran monumento, el pedestal está lleno de flores y velas. Es un grupo, me hicieron pensar en náufragos que llegasen a una isla. Pero lo que impresiona más que las estatuas es el vacío alrededor. El vacío que rodea las estatuas. Enfrente un cartel anuncia que van a hacer allí un museo. Apoyados en el cartel había unos chicos fumando. Seguí por aquí, por Zamenhofa, y poco después aquí, en el cruce con Mila, vi otro monumento. Este no tiene figuras, es solo una piedra negra, quemada, de las ruinas del gueto. Han grabado muchos nombres en ella. Debajo están los últimos que resistieron, allí cayeron y allí están enterrados. Pero la piedra está como perdida entre esas torres de apartamentos. En una de las fotos, la calle Mila parecía la más alegre del mundo, una calle llena de niños. Hoy no hay nada (Mayorga, 2010, pp. 350-351).

Pero no solo los acontecimientos se inscriben en el espacio, sino que asimismo y de manera deliberada se pueden producir, conservar y/o preservar esas "marcas de memoria" en el espacio. Esto nos lleva a la segunda dimensión antes propuesta. El ejemplo quizás más claro de esta dimensión refiere a la monumentalización y/o musealización de la memoria. Con esto me estoy refiriendo a la búsqueda, por parte de un grupo, por conservar ciertos lugares que poseen unas "marcas" específicas, y esta conservación muchas veces lleva a la musealización de ese lugar (se podrían poner varios ejemplos de este tipo, tomando el caso de la musealización de los campos de concentración y exterminio producidos por el régimen nazi).

Así también puede darse el caso de que se produzca, ex profeso, algún tipo de marcación en un lugar que necesariamente no debe tener una vinculación con el lugar del acontecimiento (sobre todo podríamos pensar aquí en la relación de los monumentos con el espacio). Esta forma en que la memoria se relaciona con el espacio nos pone frente a un modo de crear "permanencias" de manera "intencional". Habría, por lo tanto, que agregar un matiz a la idea de permanencia de Harvey: las permanencias 
no son solo contingentes, sino que también pueden ser el resultado de una búsqueda intencional con unos intereses específicos.

Por último, propongo un tercer nivel de análisis donde se haga hincapié en los procesos de memoria que se disparan en el espacio. La experiencia que se puede hacer del lugar que otrora ocupara el gueto se constituye también a partir de lo que se piensa, experimenta y recuerda estando en ese espacio y tiempo determinados. Los tiempos subjetivos ${ }^{8}$ de quien lo está vivenciando se abren paso entre el espacio y tiempo de ese lugar en particular. Esta temporalidad subjetiva trae aparejada consigo otros tiempos y espacios que no necesariamente coinciden con el lugar donde se está. A su vez, ese sujeto particular que recuerda está inextricablemente imbuido por la memoria colectiva que lo atraviesa, le hace recordar unas cosas y no otras y le hace recordar de un modo y no de otro 9 .

Pensemos en el personaje principal de la obra de Mayorga. Blanca recorre la ciudad actual de Varsovia y recuerda el gueto (recuerda el gueto con recuerdos que no son "suyos" desde un sentido cronológico). Asimismo, el recuerdo de Blanca está permeado por su historia personal (sus mudanzas, la muerte de su hija, la relación con su marido). Aunque Blanca recorre las calles de Varsovia muchísimas décadas después de que el gueto desapareciera casi por completo, ella tiene/hace una experiencia del gueto. Hay una experiencia de lo perimido que solo puede hacerse en el espacio. Cuando uno se encuentra en lugares con una profusión de marcas significativas -como bien podría serlo el espacio que otrora ocupó el gueto de Varsovia-, estos lugares tienen la capacidad de "convocar" otros espacios y tiempos que hacen referencia tanto a la historia personal del sujeto que recuerda como a la historia social del espacio.

8 Habria que tener presente también que la estructura psíquica está atravesada por diferentes temporalidades; es decir, teniendo en cuenta la primera tópica freudiana, analíticamente nos encontramos con la triada consciente, preconsciente e inconsciente. Solo la dimensión consciente se corresponde con el tiempo y el espacio kantiano; la preconsciente se constituye con esas categorías pero puede carecer o deshacerse de ellas para determinados procesos; y finalmente, el inconsciente funciona prescindiendo de ellas por completo, ya que este no tiene "tiempo". Todo puede suceder alli de manera conjunta; pasado, presente y futuro se indeterminan.

9 "Remembering, after all, is more than just a spontaneous personal act. It is also regulated by unmistakably social rules of remembrance that tell us quite specifically what we should remember and what we can or must forget" (Zerubavel, 1996, p. 286). "The notion of a collective memory implies a past that is not only commonly shared but also jointly remembered (that is 'co-memorated'). By helping ensure that an entire mnemonic community will come to remember its past together, as a group, society affects not only what and who we remember but also when we remember it" (Zerubavel, 1996, p. 294). 
$\mathrm{Al}$ reflexionar sobre estos lugares tomando el marco conceptual que se abre a partir de los desarrollos en torno al espacio-tiempo relacional, se puede profundizar justamente sobre esos espacios y tiempos otros que el sujeto trae al aquí y al ahora de ese lugar. Pero, a su vez, también permite dar cuenta de los espacios y tiempos otros que ese mismo lugar evoca y los tiempos por los que ese lugar ha sido atravesado ${ }^{10}$.

Lo interesante en relación con este último aspecto de la triada es poner en evidencia el importante papel que tienen los procesos de rememoración —individuales y colectivos- en la conformación de un lugar determinado. Esta dimensión de análisis nos permite, entonces, aproximarnos a Varsovia como un lugar constituido por una multiplicidad de redes de relaciones contemporáneas y anacrónicas.

El objetivo de proponer dicha triada ha sido un intento por desagregar - aunque solo sea de manera analítica - las distintas dinámicas en las que la memoria se anuda en el espacio.

\section{En Varsovia hay muchos mundos posibles}

Teniendo como marco de referencia la triada que propuse en el apartado anterior, compuesta por la memoria inscrita materialmente en el espacio, la construcción y/o conservación "intencional" de ciertas memorias en el espacio y la memoria - subjetiva y/o colectiva- asociada a la vivencia del lugar, retomaré algunos desarrollos del filósofo Walter Benjamin para profundizar, sobre todo, en relación con el tercer aspecto.

Para comenzar, habría que dar cuenta de que el autor berlinés ha puesto en evidencia que no todos los actos de memoria tienen la capacidad de provocar los mismos efectos. Muchas veces cuando se "hace memoria" se está meramente reproduciendo un estado de cosas dado. Esto quiere decir que no necesariamente en cada ejercicio de memoria anide la posibilidad de generar un efecto disruptivo en el presente; por tanto, la memoria puede jugar tanto un papel conservador como subversivo. La memoria, cuando es mera repetición, es "conservadora" porque aísla, es decir, impide que el pasado interactúe en/con el presente. La memoria, a su vez, también puede tener un papel altamente perturbador cuando logra situar lo que ha sido trunco (das

10 En relación con la problematización de los diferentes espacios-tiempos que un mismo lugar evoca, puede verse el artículo de Harvey (1979): "Monument and myth", donde trabaja a partir del análisis de la Basilica de Sacré-Coeur. 
Gewesene) - aquella parte del pasado que fue violentamente detenida - en el presente (das Gegenwart), trastocando al mismo tiempo tanto el presente como el pasado. De esta manera, el pasado deja de ser algo "a observar" y pasa a ser algo de lo que puede tenerse experiencia (Erfahrung).

Ahora quisiera sumar el factor espacial a esta lectura más convencional de los desarrollos de Benjamin en torno a la memoria. Se tiene experiencia de lo que ha sido trunco, en un espacio y un tiempo que ya son siempre distintos al espacio y tiempo donde el acontecimiento tuvo lugar. Es por ello que propongo pensar la experiencia del pasado - que posibilita la rememoración-como siempre diferida (algo que se percibe en un momento temporal distinto al momento en que el acontecimiento tuvo lugar) y dislocada (algo que se saca de "su lugar")"

Benjamin deja muy claro el hecho de que el pasado tal cual aconteció es irrecuperable, es pura pérdida. Cuando se tiene experiencia del espacio y tiempo del gueto desde el presente, por ejemplo, se la tiene siempre de una manera diferida y dislocada. Lo que ha sido trunco pervive de un modo diferente, en un espacio y tiempo otro. Además de este emerger de lo pasado como siempre diferido y dislocado, lo rememorado, al irrumpir, produce un desajuste en el presente. Trataré de explicitar esto a continuación.

La irrupción de lo que ha sido trunco es siempre una irrupción situada; es decir, el pasado cobra visibilidad enlazado con un lugar particular y no con otro. Existe una relación particular entre el espacio material y el recuerdo. Hay algo en ese instante (en ese espacio y tiempo determinados) que nos evoca tal recuerdo y no otro. Pero no solo el recuerdo irrumpe siempre situado, sino que además siempre transfigura el espacio donde irrumpe, lo cambia, lo transforma ${ }^{12}$.

El acto de rememoración acontece en un espacio y tiempo que necesariamente va a transformar. El vínculo entre la memoria y el espacio en Benjamin podría pensarse de la siguiente manera: lo que acontece como rememoración nos descentra, nos

11 A diferencia, las "políticas de la memoria" que procuran producir una memoria ordenada, una memoria que se asocie a unos lugares puntuales y no a otros; en un intento, quizás meramente en vano, de quitarle a la memoria esta capacidad de diferir y dislocar.

12 También podría darse el caso que el lugar donde emerge el recuerdo no tenga absolutamente nada que ver con el proceso de rememoración, pero el recuerdo que acontece sigue igualmente transfigurando el lugar donde emerge. 
mueve del lugar en donde el recuerdo nos asaltó (o hace del lugar en el que estamos otro lugar lleno de referencias/afinidades/correspondencias/citas que previamente no estaban allí) porque introduce en el espacio otro(s) espacio(s), otro(s) tiempo(s).

Propongo, entonces, aproximarnos al proceso de rememoración como una acción que siempre sitúa (da-lugar a la ausencia) y que, a la vez, corre de lugar (disloca el presente). Pero, ¿qué es lo que sitúa? ¿A qué se le da-lugar y a qué se lo corre?

Los hechos pasan a ser lo que ahora mismo nos sobrevino, constatarlos es la tarea del recuerdo, y en efecto, el despertar es la instancia ejemplar del recordar [...] Lo que quiere decir Proust cuando reordena mentalmente los muebles en la duermevela matinal (Benjamin, 2005, p. 394) ${ }^{13}$.

Benjamin habla de la rememoración como una acción análoga al acto del despertar. La rememoración acontece en ese instante donde lo real aún está corrido, donde el mundo pretérito (el mundo de los sueños) aún permanece solapado en el presente (el mundo de la vigilia). La rememoración distorsiona la imagen del presente, desorganiza el presente. En esta imagen que nos ofrece Benjamin, a través de Proust, creo que puede percibirse claramente la íntima relación entre la rememoración y el espacio: "Dónde estaba, todo daba vueltas a mi alrededor en la oscuridad, la casa, los países, los años” (Proust, citado en Benjamin, 2005, p. 408).

Cuando uno despierta, la realidad de la vigilia está corrida/movida/alterada por presencias extemporáneas y foráneas. El mundo de la vigila se conmueve por presencias anacrónicas y "fuera de lugar". Si el recordar tiene la estructura del despertar, es por el gesto de superponer un mundo que "no es" (que no llegó a tener el estatuto de ser) sobre un mundo que concebimos como único. Esta superposición -fuera de tiempo y fuera de lugar-produce una distorsión altamente desestructurante para el sujeto que la experimenta. Lo interesante de este planteo no solo es el momento de disolución de la realidad que se consideraba "sólida" y, por tanto, imperturbable, sino que esta

13 Dado que la traducción del alemán al español de este pasaje puede resultar algo confusa, cito aquí la versión original en alemán: "Und in der Tat ist Erwachen der exemplarische Fall der Erin(n)erns: der Fall, in welchem es uns glückt, des Nächsten, Banalsten, Naheliegendsten und zu erinnern. Was Proust mit dem experimentierenden Umstellen der Möbel im morgendlichen Halbschlummer meint, Bloch als Dunkel des gelebten Augenblicks erkennt, ist nichts anderes als was hier in der Ebene des Geschichtlichen, und kollektiv, gesichert werden soll. Es gibt Noch-nicht-bewusstes-Wissen vom Gewesenen, dessen Förderung die Struktur des Erwachens hat" [K 1, 2] (Benjamin, 1982, p. 491). 
desorientación espacial y temporal oficia también como umbral que posibilita la construcción de nuevas constelaciones de espacio-tiempo.

Al personaje de Blanca se lo podría pensar como un flâneur que en su deriva por Varsovia erige "topográficamente la ciudad diez y cien veces" (Benjamin, 2005, p. 110). Hay una, diez y cien ciudades en una misma ciudad. Hay tantas ciudades en Varsovia como espacios donde queramos comenzar a construirla. El flâneur es el que camina-construye estas ciudades. "Solo en apariencia es uniforme la ciudad" (Benjamin, 2005, p. 115). Diferentes ciudades habitan en una misma ciudad. El contorno, la forma de la ciudad cambia constantemente:

Blanca: ¿Qué ciudad es esta?

Samuel: No sé.

Blanca: Esa ciudad no existe.

Samuel: ¿ ?

Blanca: No hay ninguna ciudad así, ni la había entonces... Unter den Linden... Boulevard Saint Germain... Campo di Fiori...

Samuel: ¿Puede leer esas letritas?

Blanca: No puedo leerlas. Pero fíjese en la forma de las calles. Caminas por la Gran Vía de Madrid y llegas al Castillo de Praga. Cruzas la Plaza de Restauradores y estás en Piazza Navona. ¡Una brújula, eso es lo que tiene en la mano ese hombre! (Mayorga, 2010, p. 353).

Este mapa es producto de la experiencia personal de un sujeto. Mapas subjetivos, constelaciones de espacios-tiempos, "cosas que estaban separadas aparecen juntas". Mapas que representan un lugar imposible, pero un espacio posible. Es un espacio relacional. Un mapa subjetivo. Un mapa hecho de relaciones. El mapa personal de cada uno. Un mapa de experiencias, un mapa que subvierte la inmutabilidad de lo material.

Dubowski: Aquí pone "La Varsovia de Gombrowicz".

Deborah: Señala sobre el mapa actual lugares importantes en la vida del escritor.

Dónde escribió tal obra, dónde conoció a tal persona. La idea es componer una 
guía de mapas biográficos siguiendo los pasos de distintos hombres. Resulta asombroso comparar algunos de esos mapas. Ver cómo hombres separados por siglos eligen las mismas calles, los mismos rincones.

Dubowski: También puede suceder lo contrario. Que dos personas vivan al mismo tiempo en un mismo lugar, pero en mundos distintos (Mayorga, 2010, pp. 380-381).

Diferentes momentos históricos y sus personajes se cruzan en un mismo lugar. No solo diferentes sujetos van dejando sus marcas en el espacio, sino que esos recorridos personales, esas derivas biográficas por el mapa de la ciudad, al ser superpuestas con otras derivas de otros sujetos, empiezan a arrojar luz sobre las correspondencias: "Ver cómo hombres separados por siglos eligen las mismas calles, los mismos rincones". Mapas que en verdad son constelaciones subjetivas. Encuentros entre diferentes historias de vidas en el espacio. Pero también existe la distancia, el desencuentro en el espacio. Como bien acota Dubowski, también puede darse el caso de "que dos personas vivan al mismo tiempo en un mismo lugar, pero en mundos distintos".

Los desarrollos antes expuestos nos permiten pensar en los espacios-mundos posibles que no llegaron a ser. Esta espacialidad trunca, por un lado, nos remite al lugar social de ese sujeto que ya no existe, a la ausencia de esa singularidad, a la singularidad perdida para siempre. A su vez, nos impele a reflexionar sobre el modo en que se "hace memoria" justamente de esos espacios que no llegaron a ser. La invención de mapas subjetivos, imaginados, les da una suerte de segundo chance a esos espacios que no llegaron ser.

En los mapas subjetivos de Blanca no hay causalidad. Una ciudad no tiene por qué estar junto con la que materialmente aparece como contigua. Y las ciudades que aparecen juntas (Varsovia, Londres, Madrid...) tampoco comparten el mismo momento histórico (Varsovia en la actualidad, Londres cuando aún su hija vivía...). Los mapas subjetivos se parecen bastante a las constelaciones de las que habla Benjamin ${ }^{14}$ :

14 "El historicismo se contenta con establecer un nexo causal entre diversos momentos de la historia. Pero ningún hecho es histórico meramente por ser una causa. Habrá de serlo, póstumamente, en virtud de acaecimiento que pueden estar separados de él por milenios. El historiador que toma de aqui su punto de partida ya no deja más que la sucesión de acaecimientos le corra entre los dedos como un rosario. Coge la constelación en que su propia época ha entrado con una (época) anterior enteramente determinada. Funda asi un concepto del presente como tiempo-ahora, en que están regadas astillas del (tiempo) mesiánico" (Benjamin, s.f., p. 65). 
Blanca: Voy a tenderme ahí. Con este lápiz, quiero que marques mi silueta.

Raúl: ¿En el suelo?

Blanca: Espera, todavía no. En esta postura, no. Así. Sin tocarme. No me toques, por favor... Gracias.

Raúl: ¿Qué vas a hacer con esto?

Blanca: Un mapa.

Raúl: ¿De verdad es un mapa?

Blanca: Miras tu cuerpo y aparecen cosas. Personas, animales, palabras. Colores, fechas. Sonidos. Lugares. Ahí está Madrid. Varsovia. Londres. Cosas que estaban separadas, aparecen juntas. Cosas olvidadas, de pronto vuelven. Ahí estás tú cuando te conocí. Alba el día que nació. Alba el primer día de colegio.

Raúl: Blanca...

Blanca: Alba caminando sola por Londres. Alba el día que murió [...].

Blanca: Se podría hacer al revés. Se podría ir por el mundo dejando trozos del cuerpo (Mayorga, 2010, pp. 384-385).

En Varsovia del siglo XXI aparece el gueto, en el gueto aparece Londres. ¿Cómo dar cuenta de las presencias anacrónicas en el presente? ¿Cómo marcar la ausencia, lo ausente? ¿Cómo darle un lugar a lo ausente, que por definición no tiene lugar? ¿Cómo marcar lo que falta, el vacío? Blanca dibuja mapas subjetivos. Cartografía su espacio y tiempo únicos. El mapa de su vida como ella la ha experimentado: es el mapa de sus recuerdos. Blanca arma constelaciones, allí aparecen múltiples espacios y tiempos que cohabitan. En los mapas subjetivos el espacio absoluto se subvierte: “Ahí está Madrid. Varsovia. Londres. Cosas que estaban separadas, aparecen juntas. Cosas olvidadas, de pronto vuelven. Ahí estás tú cuando te conocí. Alba el día que nació. Alba el primer día de colegio”.

Los mapas de Blanca introducen el tiempo anacrónico, discontinuo, el tiempo de lo que ha sido trunco y que se anuda en el espacio. Blanca vive la ciudad de manera yuxtapuesta. Para Blanca, Varsovia es el resultado de un collage. Blanca modifica y hace suya la ciudad de Varsovia, introduciendo en su experiencia de la ciudad otros espacios y tiempos que no se corresponden necesariamente con Varsovia. En Varsovia 
se encuentra con acontecimientos acaecidos en otros espacios y tiempos, pero que al hacerse presentes allí modifican a la Varsovia misma, la vuelven una ciudad completamente diferente a la Varsovia cronológica. El tiempo no discurre continuo. El tiempo (los tiempos) no solo trae lo que ha sido trunco al presente, sino que en esas apariciones también aparecen otros espacios.

La superposición puede ser subjetiva (los espacios y tiempos otros que uno trae a ese lugar a partir de la experiencia personal), pero puede ser también una superposición "socialmente construida" (hay un "deber ser" de la memoria, es decir, hay circulaciones sociales de lo que en un lugar como Varsovia "se debería" recordar); a su vez hay otro tipo de experiencia de superposición que podríamos situar en el medio de estas dos, donde la superposición de espacios se realiza de manera deliberada por el sujeto ${ }^{15}$ :

Blanca: Una idea que he tenido. Para un mapa de Varsovia.

Raúl: ¿?

Blanca: Mira.

Raúl: ... ¿Por qué dos colores? Esa zona azul.

Blanca: La sombra del gueto.

Raúl: ¿?

Blanca: El espacio que ocupó el gueto. Las líneas de puntos marcan las sucesivas reducciones.

Raúl: ¿Un mapa para turistas? "Visite Varsovia. Un viaje a la depresión”.

Blanca: Un mapa para los que viven aquí. Es parte de la ciudad. Debe estar en el mapa.

Raúl: ¿Qué ha dicho el concejal?

15 Hay ejercicios artísticos/politicos muy interesantes donde se plantea dicho juego de superposición (que no refieren a superponer diferentes historias de un mismo lugar, sino a hacerlo en dos espacios completamente distintos en un mismo lugar). Esto podemos verlo en los ejercicios de dislocación que proponen la organización youarenothere.org con su forma de hacer turismo urbano mash-up (machacado). La propuesta es convertirse en "metaturistas", haciendo excursiones simultaneas a través de múltiples ciudades; por ejemplo, se ofrecen mapas para que cuando uno se encuentre en Tel Aviv, pueda simultáneamente experimentar la ciudad de Gaza, o en New York, la ciudad de Baghdad (ver http://www.youarenothere.org/). 
Blanca: Me ha hablado de ese museo que van a construir y de todos esos monumentos. Pero no se trata de museos ni de monumentos. Le he contado una idea que se me ha ocurrido esta mañana, caminando por allí.

Raúl: ¿Otra?

Blanca: Marcar la silueta del gueto en el suelo de la actual Varsovia (Mayorga, 2010, pp. 357-358).

Hacer un mapa de Varsovia con la sombra del gueto es disruptivo no solo por la obvia incomodidad que puede provocar en los habitantes de Varsovia y en los turistas el recordatorio del espacio de confinamiento, sino también por el hecho de superponer un espacio-tiempo otro - en el que "los hombres salían a cazar hombres" - que se recuerda pero que se considera perimido sobre la representación material de la ciudad actual, que como suele responder a la idea de un espacio absoluto, no debería registrar la anacronía.

Materializar la ausencia. Darle cuerpo a la ausencia. ¿Qué es lo que debe o no debe estar? ¿Qué marcas son las legítimas? ¿Qué marcas mejor esconder? Considero que de manera nada azarosa Mayorga decide hablar de la sombra del gueto. Es la presencia de lo ausente, de lo que ya no está ni existe, pero sigue teniendo presencia en el presente. El gueto ha desaparecido, pero sigue ejerciendo su influencia, las más de las veces silenciosa, sobre la ciudad de Varsovia. Por ello, hablar de la sombra del gueto sobre el mapa actual de Varsovia es una imagen más que acertada ${ }^{16}$. "Marcar la silueta del gueto", marcar la ausencia, el rastro.

\section{Reflexiones finales: ¿dónde ha quedado el gueto de Varsovia?}

Hay una pregunta que lacera constante al personaje de Blanca durante la obra: ¿dónde ha quedado el mapa del gueto de Varsovia que dibujó la niña? En esa pregunta asoma la necesidad de ver los rastros, de conservar las marcas de lo que ya no existe. Pero otras preguntas van también aflorando a lo largo de la obra: ¿dónde estaba

16 En efecto, en la actualidad hay proyectos que intentan llevar a cabo lo que el personaje de Blanca aqui plantea. En el blog de Ed. (2011, 31 octubre): "An Exercise In Memory. Walking Through the Warsaw Ghetto to Connect Place with Historical Memory", podemos encontrar cómo en un mapa 3d de la Varsovia contemporánea se superpuso el trazado del muro que contenía al gueto. 
exactamente el gueto en la ciudad? ¿De qué manera pervive aquello que ya no existe? ¿De qué modo las ruinas casi imperceptibles del gueto siguen allí? ¿Cómo se vive con la conciencia de saber que se transita por dentro y fuera de lo que en su momento fue el gueto? ¿Qué experimenta el sujeto cuando está frente a unas ruinas aparentemente ausentes? ¿Qué se considera "ruinas"? ¿De qué manera el tiempo del gueto se anuda en el espacio de la Varsovia actual? ¿Qué tipo de experiencia se puede tener hoy de todo ello? La obra de Mayorga termina con el siguiente párrafo:

La Niña entra. Elige una baldosa del suelo, la levanta; en el reverso de la baldosa hay marcas. La Niña saca un punzón y hace otra marca. Si diésemos la vuelta a todas las baldosas, las veríamos como cuadrículas de un mapa de Varsovia (Mayorga, 2010, p. 390).

El mapa está en el reverso, oculto, pero está. El mapa del gueto de Varsovia es Varsovia misma. Eso significa que la memoria del gueto pervive en la ciudad, pese a que ya no existe; es decir, pese a la desaparición casi total del gueto, su cartografía (sus marcas en el espacio) permanecen justamente allí, en la materialidad de lo ausente, en la materialidad de lo que falta. La niña del gueto sobrevivió, su nombre es Deborah. La niña se ha convertido en cartógrafa:

Deborah: Si quieres conocer un lugar, tienes que perderte en él. Si quieres comprender cómo funciona, quién manda y quién obedece, si quieres ver la frontera entre la ciudad de los señores y la de los que solo tienen su cuerpo, tienes que perderte. Nunca tomo notas, pero al volver a casa podría repetir cada uno de mis pasos. Camino lentamente, haciendo memoria, como si tuviese que contar lo que he visto a alguien que me espera. Siento que la gente me mira mal, el que camina despacio y mirando despierta sospechas [...] No basta mirar, hay que hacer memoria, lo más difícil de ver es el tiempo. Un adorno en un portal, un dibujo en una baldosa son señales a punto de perderse para siempre [...] Todo va a borrarse, la cabeza es como un mapa rodeado de agua, un papel que quiere deshacerse. Lo último que se borrará es lo que nadie podría dibujar (Mayorga, 2010, p. 389).

La violencia deja huellas, y esas huellas son las que inquietan (y las que se inquietan), las que hacen resurgir el reclamo de memoria. Los cuerpos y las ciudades llevan las 
marcas de la violencia, marcas que dan prueba de lo que la violencia ha hecho con ellos. En esas huellas habita la demanda de memoria ${ }^{17}$.

El que recuerda en el espacio y tiempo presente es el cuerpo. Las huellas aguardan inscritas en el cuerpo de las víctimas. Son huellas que están inscritas tanto en la realidad objetiva y material del cuerpo como en la realidad subjetiva -y fundamentalmente simbólica- de la estructura del sujeto. Son huellas que ciertos lugares agitan. El cuerpo marcado de Blanca, las huellas de Blanca se vuelven también las huellas de Varsovia; las huellas de Varsovia también se imprimen sobre el cuerpo de Blanca.

\section{Referencias}

Benjamin, W. (1982). Das Passagen-Werk. Gesammelte Schriften (Band v. 1). BadenBaden: Suhrkamp.

Benjamin, W. (2005). Libro de los pasajes. España: Akal.

Benjamin, W. (s.f.). Sobre el concepto de historia. En La dialéctica en suspenso. Fragmentos sobre la historia. Santiago de Chile: Arcis-Lom.

Ed. (2011, 31 octubre). Warsaw Ghetto Wall Superimposed On A 3D Map Of Warsaw. Recuperado de http://www.anexerciseinmemory.com/

Harvey, D. (1979). Monument and myth. Annals of the Assciation of American Geographers, 69(3), 362-381.

Harvey, D. (1996). Justice, nature \& the geography of difference. Cambridge: Blackwell.

Harvey, D. (2006). Space as a keyword. En Castree, N., y Gregory., D. (Eds.). A critical reader David Harvey. India: Blackwell.

Lefebvre, H. (1991). The production of space. Malasia: Blackwell.

Mayorga, J. (2010). El cartógrafo. Varsovia, 1:400.000. En Sucasas, A. y Zamora, J. A. (Eds.). Memoria, política, justicia. En diálogo con Reyes Mate (pp. 349-390). Madrid: Trotta.

17 Pero a su vez, el acto de memoria también ejerce un tipo de violencia, porque transfigura. Hay un tipo de violencia que aperturaria el camino para la rememoración (Eingedenken), y que a su vez hay una violencia que se realiza en el proceso mismo de rememoración. 
Murdoch, J. (2006). Post-structuralist geography. A guide to relational space. Gateshead: Sage Publications.

Teunissen, H. (2011). Topography of terror: Maps of the Warsaw ghetto. Recuperado de http://www.siger.org/warsawghettomaps/

Zerubavel, E. (1996). Social memories: steps to a sociology of the past. Qualitative Sociology, 19(3), 283-299. 